Article

\title{
Reducing Pull-In Voltage by Adjusting Gap Shape in Electrostatically Actuated Cantilever and Fixed-Fixed Beams
}

\author{
Darcy T. Haluzan ${ }^{1,2, *}$, David M. Klymyshyn ${ }^{1,2}$, Sven Achenbach ${ }^{1,2}$ and Martin Börner ${ }^{3}$
}

1 Department of Electrical and Computer Engineering, University of Saskatchewan, 57 Campus Drive, Saskatoon, Saskatchewan, S7N 5A9, Canada; E-Mails: david.klymyshyn@ usask.ca (D.M.K.); s.achenbach@usask.ca (S.A.)

2 TRLabs, 111-116 Research Drive, Saskatoon, Saskatchewan, S7N 3R3, Canada

3 Karlsruher Institut für Technologie, Institut für Mikrostrukturtechnik (IMT), Postfach 3640, D-76021 Karlsruhe, Germany; E-Mail: martin.boerner@kit.edu

* Author to whom correspondence should be addressed; E-Mail: darcy.haluzan@ usask.ca; Tel.: +1-306-668-9300; Fax: +1-306-668-1944.

Received: 26 May 2010; in revised form: 9 July 2010 / Accepted: 26 July 2010 /

Published: 28 July 2010

Abstract: A gap with variable geometry is presented for both cantilever beam and fixed-fixed beam actuators as a method to reduce the pull-in voltage while maintaining a required displacement. The method is applicable to beams oriented either in a plane parallel to or perpendicular to a substrate, but is most suitable for vertically oriented (lateral) beams fabricated with a high aspect ratio process where variable gap geometry can be implemented directly in the layout. Finite element simulations are used to determine the pull-in voltages of these modified structures. The simulator is verified against theoretical pull-in voltage equations as well as previously published finite element simulations. By simply varying the gap in a linear fashion the pull-in voltage can be reduced by $37.2 \%$ in the cantilever beam case and $29.6 \%$ in the fixed-fixed beam case over a structure with a constant gap. This can be reduced a further $4.8 \%$ by using a polynomial gap shape $(n=4 / 3)$ for the cantilever beam and $1.2 \%$ for the fixed-fixed beam by flattening the bottom of the linearly varying gap.

Keywords: electrostatic; actuator; gap shape; finite element; MEMS; pull-in voltage; high aspect ratio; LIGA 


\section{Introduction}

Electrostatic actuation as a method to obtain movement in microelectromechanical systems (MEMS) devices is extremely popular since this actuation method provides simplicity, fast actuation rates, very low, if not zero power consumption, and no special material requirements. These actuators are often found in the form of cantilever and fixed-fixed beams and are found in MEMS devices such as microwave variable capacitors [1,2] and switches [3], optical micro-mirrors [4] and valves for fluidics applications [5]. One of the issues with electrostatic actuation is that the actuation voltage can become quite large especially for applications requiring large deflections.

In [4], the authors describe increasing the travel range of electrostatic actuators using leveraged bending. This increases deflection, but comes at the expense of increased actuation voltage. They suggest that varying the gap distance along the length of the beam could be considered to lower actuation voltage, however what particular shapes these gaps should have is not discussed. In [6], the authors present curved electrode cantilever electrostatic actuators with large curvature $(n \geq 2)$ capable of very large deflection, but these designs require that either a dielectric insulator or stand-off bumper structures be placed between the beam and the attracting electrode. The beam must contact these structures as deflection occurs, which may not be possible or desirable. Other designs with variable geometry have been presented [7,8], but not with the intention of finding specific shapes to reduce the pull-in voltage for a given required displacement.

In this paper, the shape of the gap between the beam and the attracting electrode is controlled in order to find suitable geometries that significantly reduce the pull-in voltage, while maintaining the required displacement before pull-in. This electrostatic-structural interaction is modeled using finite element simulation and verified using theoretical equations for pull-in voltage as well as previously published pull-in voltage results from an independent simulator.

\section{Problem Description}

MEMS cantilever and fixed-fixed beam structures are shown in Figure 1. These structures exemplify the more typical planar approach and feature thin beams suspended over a ground plane. The ground plane and the beam are electrically isolated and anchored by a dielectric layer, which also determines the gap distance between the beam and the ground plane. The beam is pulled toward the ground plane by applying a bias voltage between the two. This bias voltage creates a separation of charges on the two surfaces, which results in an electrostatic force. As the voltage is increased, the beam bends stably toward the ground plane until a voltage called the "pull-in" voltage is reached. At this point, the beam collapses onto the ground plane.

Less common are vertically oriented (lateral) beams that are perpendicular to the substrate as shown in Figure 2, which can be fabricated using various micromachining technologies. Beams and gaps with high aspect ratio features are particularly well suited for realization using the LIGA (German acronym for lithography, electroplating and moulding) process [1,9]. Vertical beam structures operate under the same principles as the planar structures. One possible advantage to beams oriented in this fashion is that the profile of the gap (the "gap shape") can be easily controlled since it is a feature on the mask. This is considerably more difficult in the planar case, where complex processing steps would be required. 
Figure 1. Planar beam configurations (a) cantilever beam (b) fixed-fixed beam.

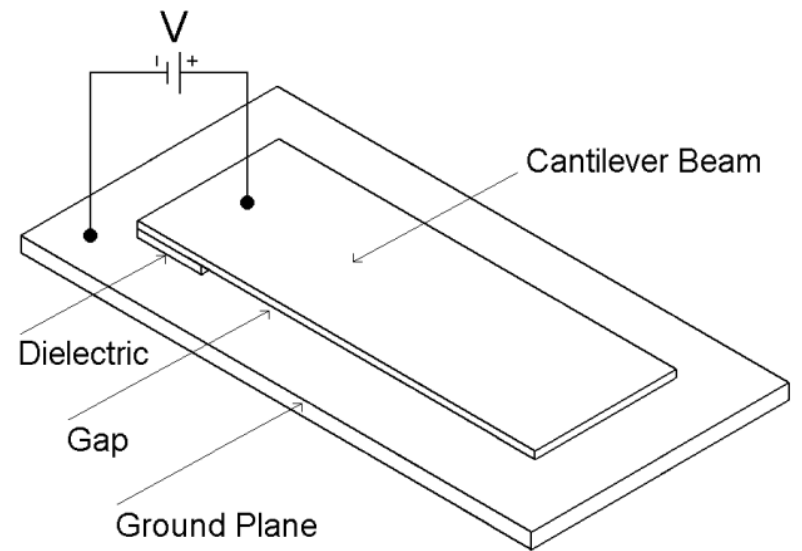

(a)

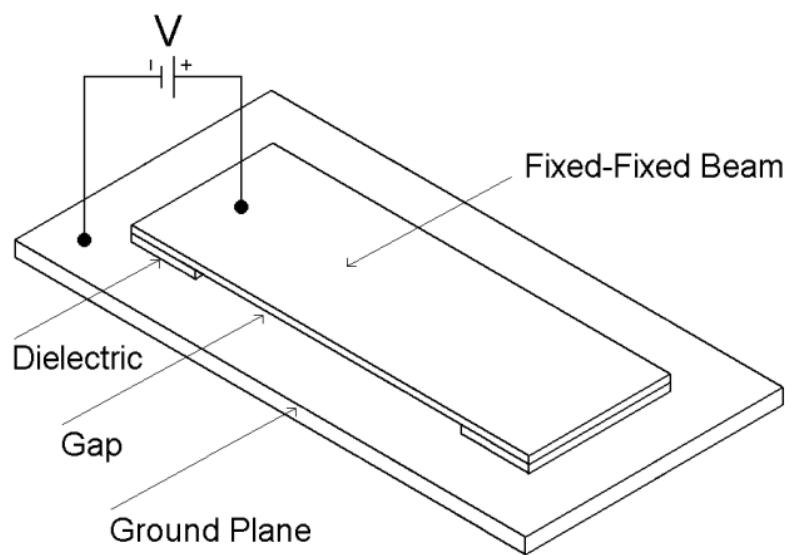

(b)

Figure 2. Vertical high aspect ratio beam configurations (a) cantilever beam (b) fixed-fixed beam.

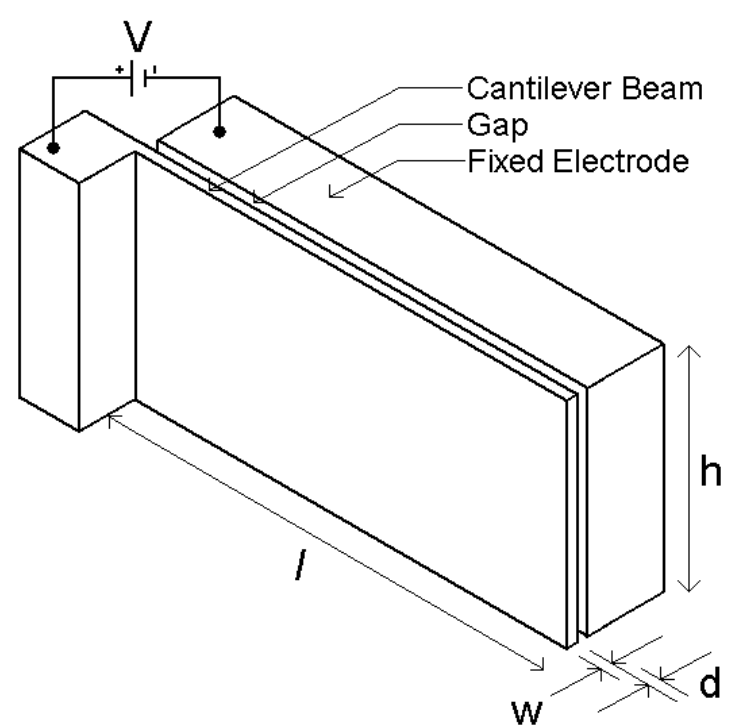

(a)

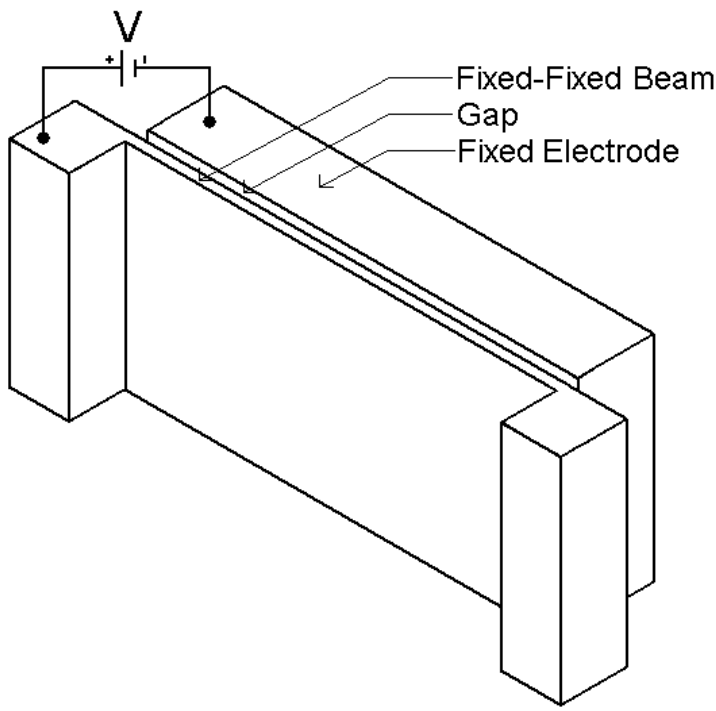

(b)

Profile views of the two different beam cases are shown in Figure 3. This study involves optimizing the shape of the gap $(d)$ as a function of the distance along the beam $(x)$, in order to minimize the pull-in voltage for a given required displacement. Beam shapes remain rectangular and as thin as possible to minimize pull-in voltage, but the profile of the gap is changed. Using finite element analysis with the software package ANSYS ${ }^{\text {TM }}$ Multiphysics [10], it is shown that by changing the gap profile, the applied voltage can be reduced and still yield the same displacement.

A scanning electron microscope (SEM) image of the tip of an electrostatically actuated vertical cantilever beam for radio frequency (RF) MEMS applications fabricated using the LIGA process is shown in Figure 4. This structure is very similar to the structure depicted in Figure 2(a) and is an example of the type of structure that can benefit from a variable profile gap to reduce the required actuation voltage. The $100 \mu \mathrm{m}$ tall nickel cantilever features a thin $7.5 \mu \mathrm{m}$ cantilever beam with an 
adjacent air gap of $1.8 \mu \mathrm{m}$. The gap features an impressive 55:1 aspect ratio and runs laterally for hundreds of microns and can be precisely controlled on a sub-micron scale as a mask feature. The pull-in voltages of RF MEMS structures similar to this have been measured previously [1] and the results are consistent with ANSYS simulations.

Figure 3. Beam profiles (a) cantilever beam (b) fixed-fixed beam.

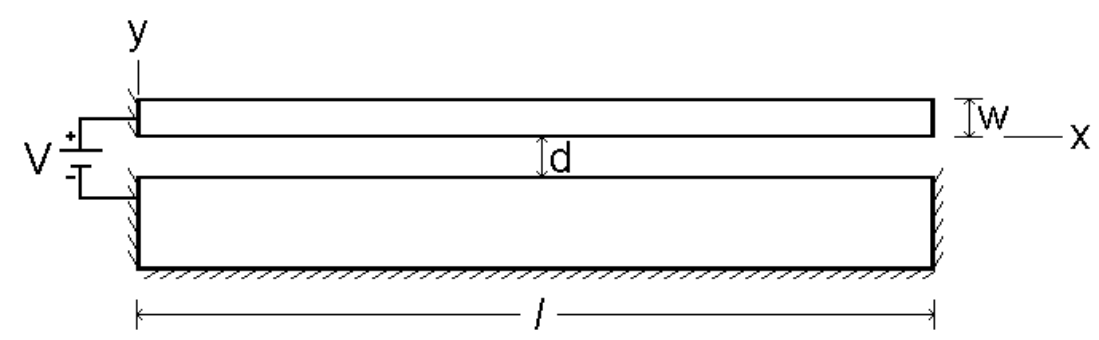

(a)

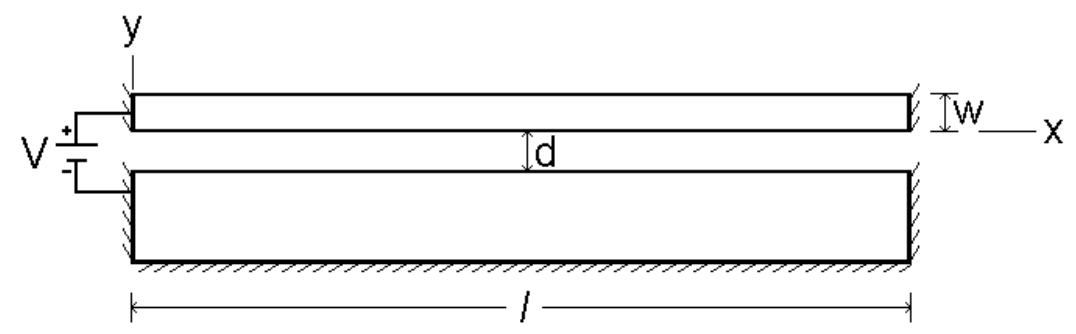

(b)

Figure 4. Tip of RF MEMS cantilever beam fabricated in nickel using the LIGA process showing narrow $(1.8 \mu \mathrm{m})$ high aspect ratio $(55: 1)$ gap. Bottom text denotes sample fabrication at IMT in Karlsruhe, Germany.

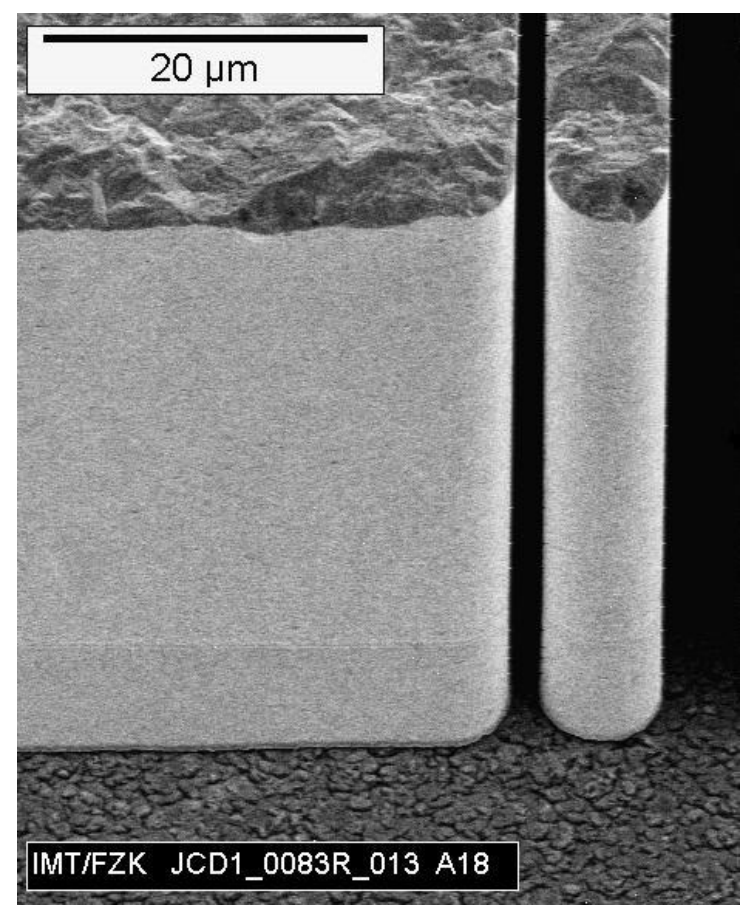

For this work, a representative problem is described and the gap shape modified to reduce the pull-in voltage. The sample problem features dimensions and material properties similar to that found 
in the RF MEMS cantilever beam shown in Figure 4. Both a cantilever and a fixed-fixed beam with common dimensions and material properties are investigated. A list of the important values is given in Table 1.

For both beam types, Young's modulus is $169 \mathrm{GPa}$, Poisson's ratio is 0.32 , the height is $100 \mu \mathrm{m}$, the length is $1,000 \mu \mathrm{m}$ and the width is $10 \mu \mathrm{m}$. The minimum gap is $1 \mu \mathrm{m}$, which is assumed to be a limitation of the process for achievable aspect ratios. The required displacement is $2 \mu \mathrm{m}$, therefore some part of the beam must move at least $2 \mu \mathrm{m}$ from its original position. For the cantilever configuration, this is likely to be the beam tip, and for the fixed-fixed configuration, the middle of the beam. This value was chosen as being a reasonable amount of deflection, suitable for many applications, but without unreasonably large actuation voltages.

Table 1. Sample problem parameters.

\begin{tabular}{lr}
\hline Young's modulus $(E)$ & $169 \mathrm{GPa}$ \\
Poisson's ratio $(v)$ & 0.32 \\
Height $(h)$ & $100 \mu \mathrm{m}$ \\
Length $(l)$ & $1,000 \mu \mathrm{m}$ \\
Width $(w)$ & $10 \mu \mathrm{m}$ \\
Min. Gap $\left(d_{\min }\right)$ & $1 \mu \mathrm{m}$ \\
Required Displacement & $2 \mu \mathrm{m}$ \\
\hline
\end{tabular}

\section{Verification}

To verify the accuracy of the finite element analysis in determining the pull-in voltage, ANSYS models were created and compared to the results presented by Osterberg [11,12] and Chowdhury [13,14]. In [11,12], multiple simulations using a two-dimensional (2D) distributed model were performed and a database of pull-in voltages was created. These results were used to create closed-form equations for the pull-in voltage of cantilever beams, fixed-fixed beams and diaphragms. In addition, for a select subset of structures, the 2D results were compared to full 3D finite element simulations using CoSolve-EM. ANSYS simulation results are compared to this subset in order to verify the accuracy of the simulator. In $[13,14]$, closed form equations for the pull-in voltages of cantilever and fixed-fixed beams were created using a VLSI on-chip interconnect capacitance model. The author claims the accuracy of these equations is better when compared to other published models. ANSYS results are also compared to the results predicted by these closed-form equations.

The closed-form equations for the pull-in voltage of cantilever and fixed-fixed beams are given in Equations 1 and 2 [11,12]. These equations correspond to the variables shown in Figure 2 and are slightly modified from [11,12] to represent the vertical rather than planar orientation of the beams. The equations are simplifications of the general case given in $[11,12]$ and assume there is no residual stress present in the beams. In addition, the equations are only valid in the small deflection regime (linear elastic mechanics) which is valid for $d / w \leq 1$ [15]. The beam equations adapted from [13] and [14] are given in Equation 3 (cantilever) and Equation 4 (fixed-fixed). Here residual stress is included and there is no limitation to small deflections. The equations apply to all structures encountered in this paper. For cases with extreme fringing fields or gaps much larger than beam width, the general equations are given in $[13,14]$. 
For short beams $(h<5 w)$, the effective modulus $\tilde{E}$ is equal to Young's modulus $E$ and the effective residual stress $\tilde{\sigma}$ is equal to the residual stress $\sigma_{0}$. For tall beams $(h \geq 5 w)$, the effective modulus becomes the plate modulus $E /\left(1-v^{2}\right)$, where $v$ is Poisson's ratio and the effective residual stress becomes $\sigma_{0}(1-v)$ [12]. All beams featured here are considered tall.

$$
\begin{aligned}
& V_{p i} \approx 0.529 \sqrt{\frac{\tilde{E} w^{3} d^{3}}{\varepsilon_{0} l^{4}\left(1+0.42 \frac{d}{h}\right)}} \\
& V_{p i} \approx 3.444 \sqrt{\frac{\tilde{E} w^{3} d^{3}}{\varepsilon_{0} l^{4}\left(1+0.42 \frac{d}{h}\right)}} \\
& V_{p i} \approx \sqrt{\frac{2 \tilde{E} w^{3} d}{8.37 \varepsilon_{0} l^{4}\left(\frac{5}{6 d^{2}}+\frac{0.19}{d^{1.25} h^{0.75}}+\frac{0.19}{d^{1.25} l^{0.75}}+\frac{0.4 w^{0.5}}{d^{1.5} h}\right)}} \\
& V_{p i} \approx \sqrt{\frac{\left\{9.35\left[\frac{\tilde{\sigma} w}{l^{2}}\right]+32.36\left[\frac{\tilde{E} w^{3}}{l^{4}}\right]\right\} \frac{d}{3}+24.35\left[\frac{\tilde{E} w}{l^{4}}\right]\left(\frac{d}{3}\right)^{3}}{1.09 \varepsilon_{0}\left(\frac{5}{6 d^{2}}+\frac{0.19}{d^{1.25} h^{0.75}}+\frac{0.4 w^{0.5}}{d^{1.5} h}\right)}}
\end{aligned}
$$

To implement this in ANSYS, the beam is created and meshed with $0.25 \mu \mathrm{m}$ rectangular structural elements of type 82 with the plane strain option turned on since the beam is tall and the plate modulus should be used. The beam and stationary electrode are surrounded by an air box which is meshed with $0.25 \mu \mathrm{m}$ rectangular electrostatic elements of type 121 . The stationary electrode does not receive mesh since the interior of it is neither a structural element nor an electrostatic element. The material properties are defined and one end of the beam is fixed. Nonlinear geometry effects are turned on using the NLGEOM command. The beam has the input voltage applied to it and the stationary electrode has $0 \mathrm{~V}$ applied to it. Structural and electrostatic physics files are written and a coupled analysis is performed using the ESSOLV macro.

The results of the comparison are given in Table 2. This data set includes both cantilever and fixed-fixed beams of varying lengths $(l)$, Poisson's ratio $(v)$, and residual stress $\left(\sigma_{0}\right)$. For all beams $E=169 \mathrm{GPa}, v=0.06$ (unless specified), $h=50 \mu \mathrm{m}, w=3 \mu \mathrm{m}$ and $d=1 \mu \mathrm{m}$. Table 2 shows that excellent agreement exists between all methods to better than $1 \%$ on average. The CoSolve-EM full 3D results are expected to be the most accurate and 2D ANSYS results agree to within $0.76 \%$ on average. This verifies that $2 \mathrm{D}$ simulations are sufficiently accurate for this work. In 2D simulations, the fringing effects at the ends of the beam are included, but the effects along the length of the beam are neglected. This makes 2D FEM analysis valid for tall beams where fringing fields are negligible. For short beams, 3D FEM analysis would likely be more accurate. 
Table 2. Comparison of 2D finite element analysis with results presented in $[11,13,14]$.

\begin{tabular}{|c|c|c|c|c|c|c|c|}
\hline Beam Description & $\begin{array}{c}\text { V }_{\text {PI }} \\
\text { 3D- } \\
\text { FEM } \\
{[11]} \\
\end{array}$ & $\begin{array}{c}\mathrm{V}_{\mathrm{PI}} \\
\text { closed- } \\
\text { form } \\
{[11]} \\
\end{array}$ & $\begin{array}{c}\mathrm{V}_{\mathrm{PI}} \\
\text { closed- } \\
\text { form } \\
{[13,14]} \\
\end{array}$ & $\begin{array}{c}\text { V } \\
\text { 2D- } \\
\text { FEM } \\
\text { [current] }\end{array}$ & $\begin{array}{c}\Delta \% \\
\text { between } \\
\text { 2D-FEM and } \\
\text { 3D-FEM [11] }\end{array}$ & $\begin{array}{c}\Delta \% \\
\text { between } \\
\text { 2D-FEM and } \\
\text { closed-form [11] }\end{array}$ & $\begin{array}{c}\Delta \% \\
\text { between } \\
\text { 2D-FEM and } \\
\text { closed-form }[13,14]\end{array}$ \\
\hline \multicolumn{8}{|l|}{ Cantilever Beams } \\
\hline$l=100 \mu \mathrm{m}$ & 38.2 & 37.9 & 37.84 & 37.85 & 0.92 & 0.13 & 0.03 \\
\hline$l=100 \mu \mathrm{m}, v=0.32$ & 39.8 & 39.9 & 39.86 & 39.90 & 0.25 & 0.00 & 0.10 \\
\hline$l=150 \mu \mathrm{m}$ & 16.9 & 16.8 & 16.83 & 16.85 & 0.30 & 0.30 & 0.12 \\
\hline Cantilever Average & & & & & 0.49 & 0.14 & 0.08 \\
\hline \multicolumn{8}{|l|}{ Fixed-Fixed Beams } \\
\hline$l=250 \mu \mathrm{m}, \sigma=0$ & 40.1 & 39.5 & 39.60 & 39.56 & 1.35 & 0.15 & 0.10 \\
\hline$l=250 \mu \mathrm{m}, \sigma=0, v=0.32$ & 41.2 & 41.5 & 41.72 & 41.70 & 1.21 & 0.48 & 0.05 \\
\hline$l=250 \mu \mathrm{m}, \sigma=100 \mathrm{MPa}$ & 57.6 & 56.9 & 57.40 & 57.35 & 0.43 & 0.79 & 0.09 \\
\hline$l=250 \mu \mathrm{m}, \sigma=-25 \mathrm{MPa}$ & 33.6 & 33.7 & 33.71 & 33.50 & 0.30 & 0.59 & 0.62 \\
\hline$l=350 \mu \mathrm{m}, \sigma=0$ & 20.3 & 20.2 & 20.20 & 20.19 & 0.54 & 0.05 & 0.05 \\
\hline$l=350 \mu \mathrm{m}, \sigma=100 \mathrm{MPa}$ & 35.8 & 35.4 & 35.91 & 35.71 & 0.25 & 0.88 & 0.56 \\
\hline$l=350 \mu \mathrm{m}, \sigma=-25 \mathrm{MPa}$ & 13.7 & 13.8 & 13.71 & 13.42 & 2.04 & 2.75 & 2.12 \\
\hline Fixed-Fixed Average & & & & & 0.88 & 0.81 & 0.51 \\
\hline Overall Average & & & & & 0.76 & 0.61 & 0.38 \\
\hline
\end{tabular}

\section{Constant Gap Configurations}

\subsection{Minimum Size Constant Gap Case}

The first scenario investigated is the case with the gaps as small as possible. This is performed in order to see how large the displacement is with small gaps as well as the magnitude of the pull-in voltage. This case is shown in Figure 3 with $d(x)=d_{\min }=1 \mu \mathrm{m}$. The remaining parameters are given in Table 1. The displacements produced and the corresponding pull-in voltages are shown in Table 3.

Table 3. Minimum constant gap case results (small displacement).

\begin{tabular}{lcccc}
\hline $\begin{array}{l}\text { Beam } \\
\text { Configuration }\end{array}$ & $\mathbf{V}_{\text {PI }}[\mathbf{V}]$ & $\begin{array}{c}\text { Maximum } \\
\text { Displacement } \\
{[\boldsymbol{\mu m}]}\end{array}$ & $\begin{array}{c}\mathbf{V}_{\text {PI }}[\mathbf{V}] \text { Osterberg } \\
\text { Closed-form } \\
{[\mathbf{1 1 , 1 2}]}\end{array}$ & $\begin{array}{c}\mathbf{V}_{\text {PI }}[\mathbf{V}] \text { Chowdhury } \\
\text { Closed-form } \\
{[\mathbf{1 3 , 1 4}]}\end{array}$ \\
\hline Cantilever & 2.44 & 0.44 & $2.43(0.41 \%)$ & $2.44(0.00 \%)$ \\
Fixed-Fixed & 15.77 & 0.39 & $15.85(0.50 \%)$ & $15.85(0.50 \%)$ \\
\hline
\end{tabular}

For these and all subsequent models, an initial element size of $0.5 \mu \mathrm{m}$ was used. This was chosen since, in general, the models increase in size over the verification case. From the results, we can see that the pull-in voltages are quite low, but so are the obtained displacements. These results agree very well with the pull-in voltage equations. The maximum difference is $0.41 \%$ in the cantilever case and $0.50 \%$ in the fixed-fixed case. 


\subsection{Required Displacement Constant Gap Case}

In this section the gap is increased, but the value kept constant until the required displacement of $2 \mu \mathrm{m}$ is achieved before pull-in. This case is also depicted in Figure 3 with $d(x)$ equal to the constant value required for $2 \mu \mathrm{m}$ of stable deflection before pull-in. The results are shown in Table 4 . With both beam configurations the required $2 \mu \mathrm{m}$ of stable deflection is achieved, but as expected, the pull-in voltage has increased dramatically over the minimum gap case. These results also agree well with the theoretical equations with a maximum difference of $1.24 \%$ for the cantilever case and $1.75 \%$ for the fixed-fixed case. The beam displacement profiles are shown in Figure 5. The results of this constant gap case are compared against the results obtained by varying the gap shape to determine the extent of the decrease in pull-in voltage.

Table 4. Constant gap case results (required $2 \mu \mathrm{m}$ displacement).

\begin{tabular}{lcccc}
\hline $\begin{array}{l}\text { Beam } \\
\text { Configuration }\end{array}$ & $\mathbf{V}_{\text {PI }}[\mathbf{V}]$ & $\begin{array}{c}\text { Gap Width }(\boldsymbol{d}) \\
{[\boldsymbol{\mu m}]}\end{array}$ & $\begin{array}{c}\mathbf{V}_{\text {PI }}[\mathbf{V}] \text { Osterberg } \\
\text { Closed-form [11,12] }\end{array}$ & $\begin{array}{c}\mathbf{V}_{\text {PI }} \text { [V] Chowdhury } \\
\text { Closed-form [13,14] }\end{array}$ \\
\hline Cantilever & 23.66 & 4.56 & $23.53(0.55 \%)$ & $23.37(1.24 \%)$ \\
Fixed-Fixed & 178.26 & 4.99 & $175.20(1.75 \%)$ & $175.43(1.61 \%)$ \\
\hline
\end{tabular}

Figure 5. Constant gap beam displacement profiles (a) cantilever (b) fixed-fixed.

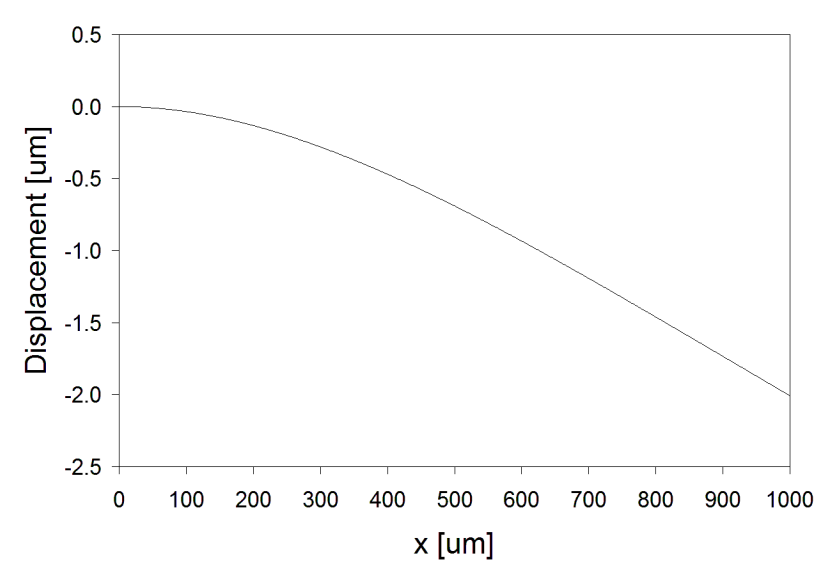

(a)

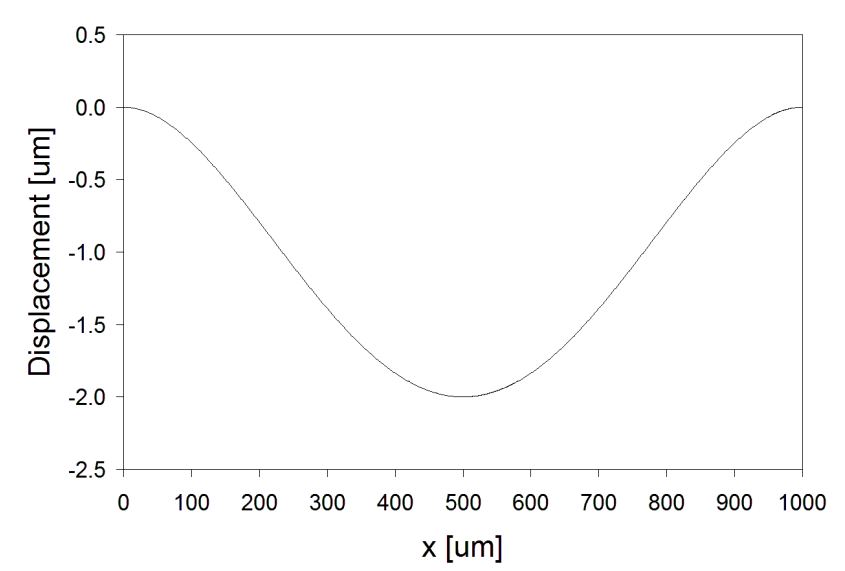

(b)

\section{Linear Gap Configurations}

\subsection{Linearly Varying Gap Case}

The case with a linear variation in gap width is shown in Figure 6. For the cantilever, the equation for the shape of the gap is $y=-\left(d_{\max } / l\right) x$ except for the region where this yields gaps smaller than $d_{\min }$. In this region the gap is set to $d_{\text {min }}$. The fixed-fixed beam is similar except that the maximum gap occurs at the $x=l / 2$ point. 
Figure 6. Linearly varying beam profiles (a) cantilever (b) fixed-fixed.

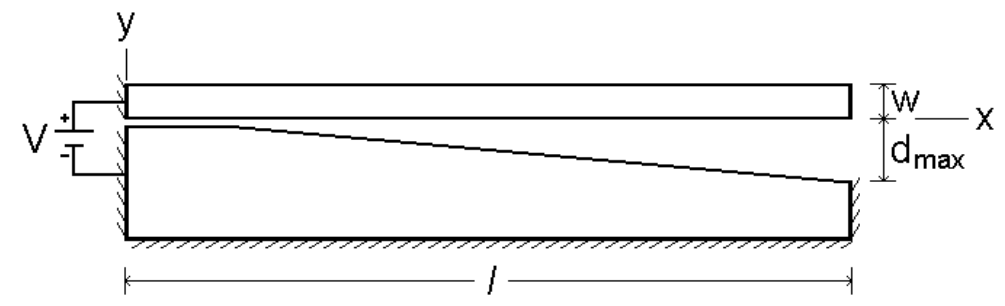

(a)

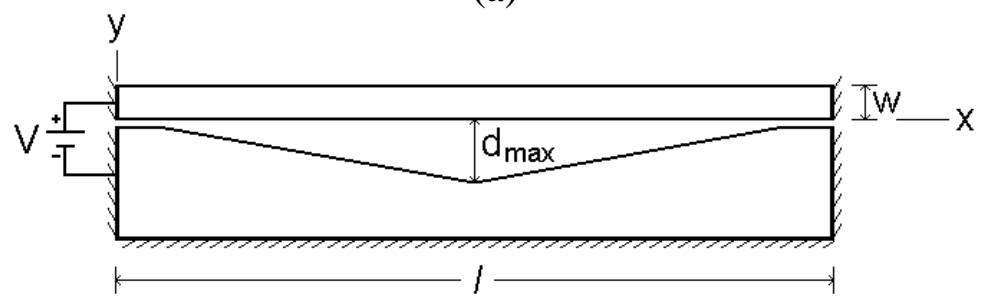

(b)

The results for this case with $2 \mu \mathrm{m}$ of stable deflection before pull-in are listed in Table 5. Clearly if the gap varies in a linear fashion, the actuation voltage can be made significantly smaller and still achieve $2 \mu \mathrm{m}$ of deflection. For the cantilever configuration, the voltage is $62.8 \%$ of the constant gap case and for the fixed-fixed configuration, the voltage is $70.4 \%$ of the constant gap case. For the fixed-fixed beam, a larger increase in gap width over the constant gap case is required to maintain the same deflection, therefore the voltage reduction is not as large as the cantilever case. The beam displacement profiles are shown in Figure 7 along with the profiles from the constant gap case. In both cantilever and fixed-fixed beam cases, the linear gap shape causes a larger average deflection along the length of the beam.

Table 5. Linearly varying gap case results (required $2 \mu \mathrm{m}$ displacement).

\begin{tabular}{llc}
\hline $\begin{array}{l}\text { Beam } \\
\text { Configuration }\end{array}$ & $\mathbf{V}_{\text {PI }}[\mathbf{V}]$ & $\begin{array}{c}\text { Gap Width }\left(\boldsymbol{d}_{\text {max }}\right) \\
{[\boldsymbol{\mu m}]}\end{array}$ \\
\hline Cantilever & 14.86 & 4.84 \\
Fixed-Fixed & 125.48 & 6.57 \\
\hline
\end{tabular}

Figure 7. Linear gap beam displacement profiles (a) cantilever (b) fixed-fixed.

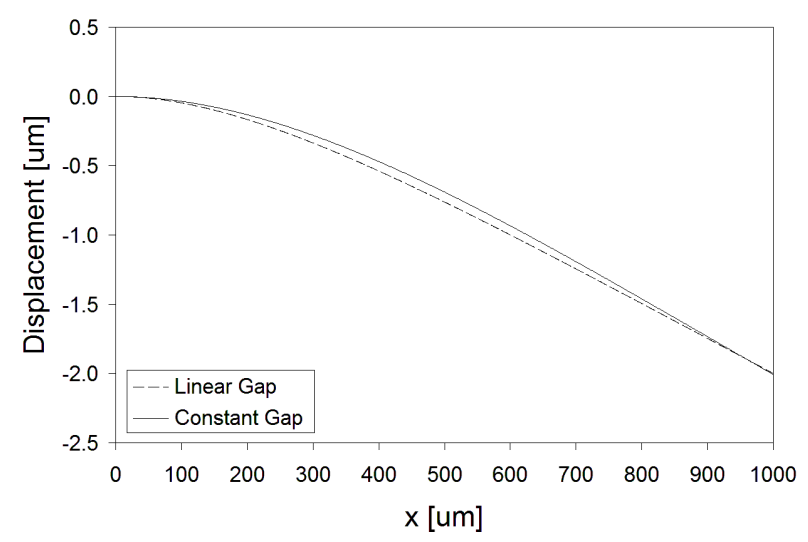

(a)

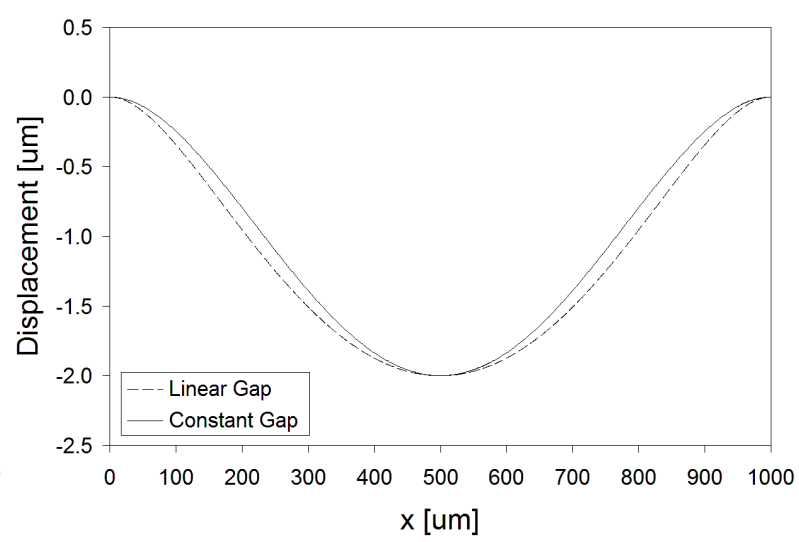

(b) 


\subsection{Linearly Varying Gap with Flattened Bottom Case}

For the fixed-fixed beam, the linearly varying gap case was modified to include a flattened bottom in an attempt to further reduce the pull-in voltage. The beam profile for this case is shown in Figure 8 .

Figure 8. Linearly varying beam profile with flattened bottom.

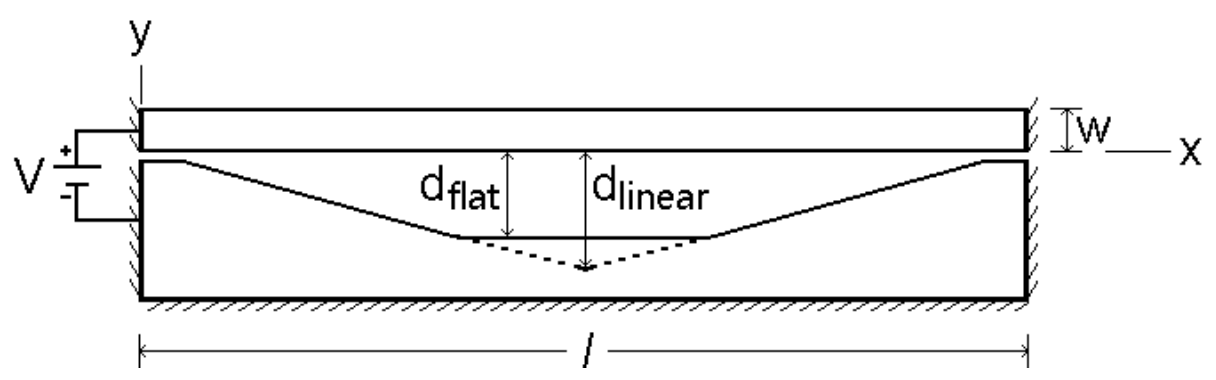

For each value of $d_{\text {flat }}$, the value of $d_{\text {linear }}$ was varied until $2 \mu \mathrm{m}$ of stable deflection was obtained before pull-in. The value of $d_{\text {flat }}$ was increased starting from $y=-6.57 \mu \mathrm{m}$, which is the value of $d_{\max }$ from the previous case. The results are shown in Table 6 with the first row being the value from the previous case. The "percentage of triangle removed" column gives the amount the triangle bottom was flattened and is defined as $\left(\left(d_{\text {linear }}-d_{\text {flat }}\right) / d_{\text {linear }}\right) \times 100 \%$. These results are plotted in Figure $9(\mathrm{a})$. From Figure 9(a), it is clear that a further reduction in pull-in voltage is possible with a minimum value of $123.94 \mathrm{~V}$, which corresponds to a flat distance of $5.20 \mu \mathrm{m}$ and a linear distance of $6.65 \mu \mathrm{m}$. This ratio results in a triangle with approximately the lower $22 \%$ flattened. The beam displacement profile for the minimum voltage case $\left(d_{\text {flat }}=5.20 \mu \mathrm{m}\right)$ is shown in Figure $9(\mathrm{~b})$. The displacement of the beam in this case is very similar to the linear gap case but with a slight reduction in average beam displacement which is due to the increase in $d_{\text {linear }}$.

Table 6. Linearly varying gap with flattened bottom case results (required $2 \mu \mathrm{m}$ displacement).

\begin{tabular}{cccc}
\hline $\begin{array}{c}\text { Flat Distance } \\
\left(\boldsymbol{d}_{\text {flat }}\right)[\boldsymbol{\mu m}]\end{array}$ & $\begin{array}{c}\text { Linear Distance } \\
\left(\boldsymbol{d}_{\text {linear }}\right)[\boldsymbol{\mu m}]\end{array}$ & $\begin{array}{c}\text { \% Of Triangle } \\
\text { Removed [\%] }\end{array}$ & $\mathbf{V}_{\mathbf{P I}}[\mathbf{V}]$ \\
\hline 6.57 & 6.57 & 0.0 & 125.48 \\
6.00 & 6.57 & 8.7 & 125.12 \\
5.50 & 6.59 & 16.5 & 124.41 \\
5.25 & 6.63 & 20.8 & 124.02 \\
5.20 & 6.65 & 21.8 & 123.94 \\
5.15 & 6.66 & 22.7 & 123.97 \\
5.125 & 6.67 & 23.2 & 123.98 \\
4.99 & 6.75 & 26.1 & 124.21 \\
4.875 & 6.85 & 28.8 & 124.74 \\
\hline
\end{tabular}


Figure 9. Results for flattened bottom case (a) pull-in voltage (b) beam displacement profile $\left(d_{\text {flat }}=5.20 \mu \mathrm{m}\right)$.

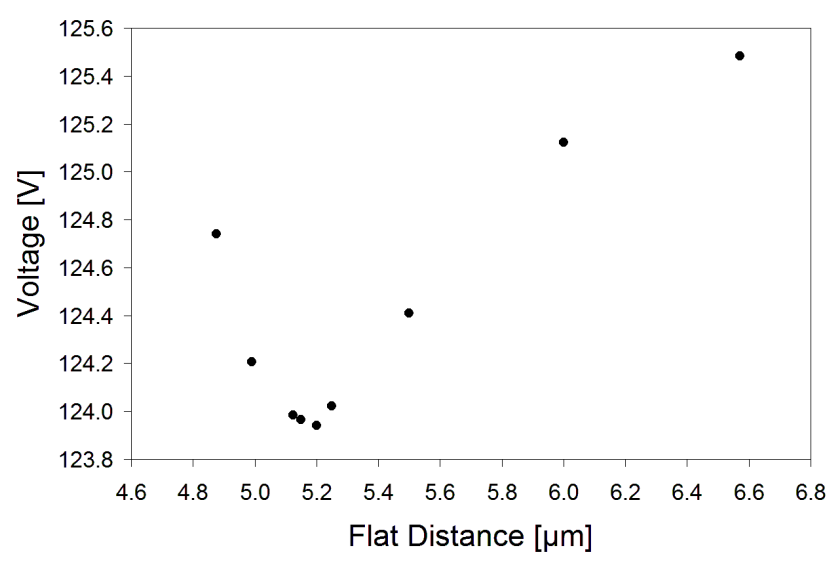

(a)

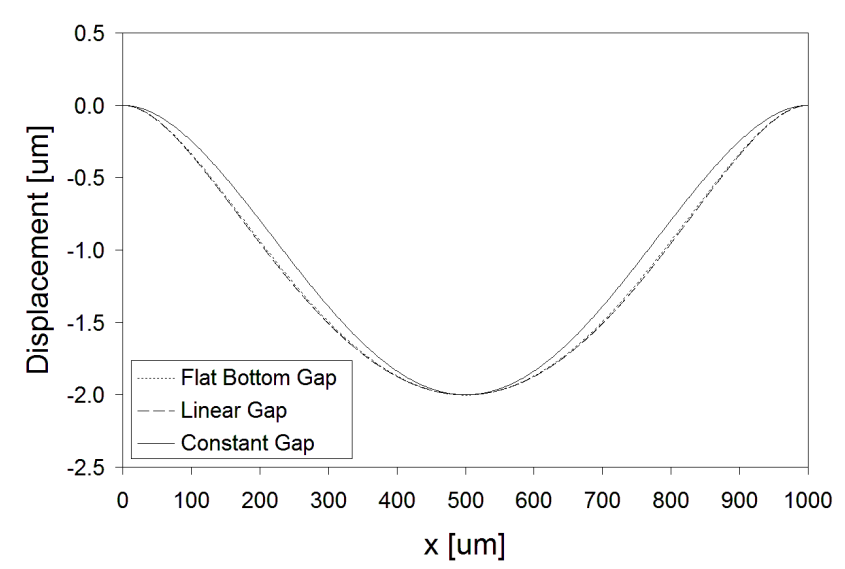

(b)

\section{Polynomial Gap Configurations}

To further reduce the pull-in voltage a gap shape with an $n^{\text {th }}$-order polynomial function was investigated. The gap shape varies according to Equation 5 for cantilever beams and Equation 6 for fixed-fixed beams. These equations hold except for the regions where this yields gaps smaller than $d_{\text {min }}$. In these regions the gap is set to $d_{\min }$. For the fixed-fixed case the equation is valid until the $x=l / 2$ point and then the gap profile is mirrored at $x=l / 2$.

$$
\begin{gathered}
d(x)=d_{\max }\left(\frac{x}{l}\right)^{n} \\
d(x)=d_{\max }\left(\frac{x}{l / 2}\right)^{n}
\end{gathered}
$$

The polynomial gap cases are shown in Figure 10 with $n>1$. In ANSYS these curves were implemented as piecewise linear curves with the $x$-axis divided into pieces of $10 \mu \mathrm{m}$ length. For both beam cases, the order $(n)$ was varied and the effect on the pull-in voltage was observed. Table 7 lists pull-in voltages with the corresponding values of $n$ for the cantilever beam and fixed-fixed beam. This data is plotted in Figure 11.

In Table 7 and Figure 11, the values for the linearly varying gap cases are included as well, which corresponds to $n=1$. For the cantilever beam case, from Figure 11(a), we can see that the pull-in voltage is smallest at a value of approximately $n=1.3333$. At this point, the pull-in voltage is lowered by $4.8 \%$ over the linearly varying gap case, and reaches a minimum at $14.14 \mathrm{~V}$. Values of $n>1$ give rise to lower pull-in voltages than the $n=1$ case up until approximately $n=1.6$ where the values become larger than the $n=1$ case. For the fixed-fixed beam case as shown in Figure 11(b), values of $n \neq 1$ give rise to higher pull-in voltages than the $n=1$ case. For the cantilever beam case, the beam displacement profiles are shown in Figure 12. Three different beam profiles are shown, based on three different gap shapes. The deflection based on the polynomial gap shape is similar to the deflection based on the linear gap shape except the polynomial gap shape has a slightly higher average deflection. 
Figure 10. Polynomial beam profiles (a) cantilever beam (b) fixed-fixed beam.

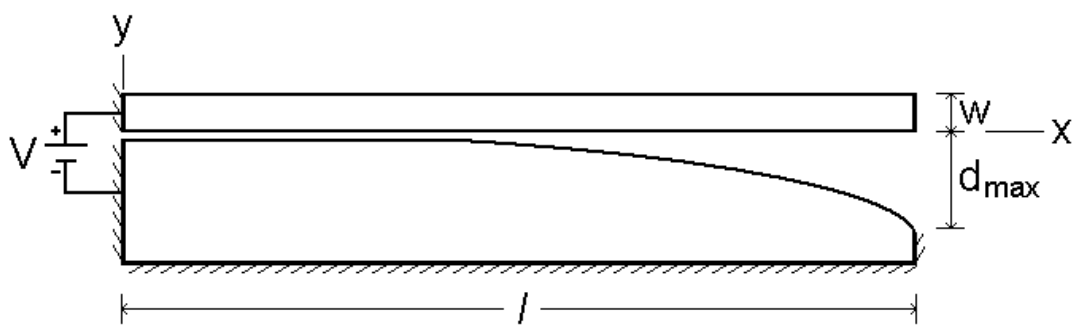

(a)

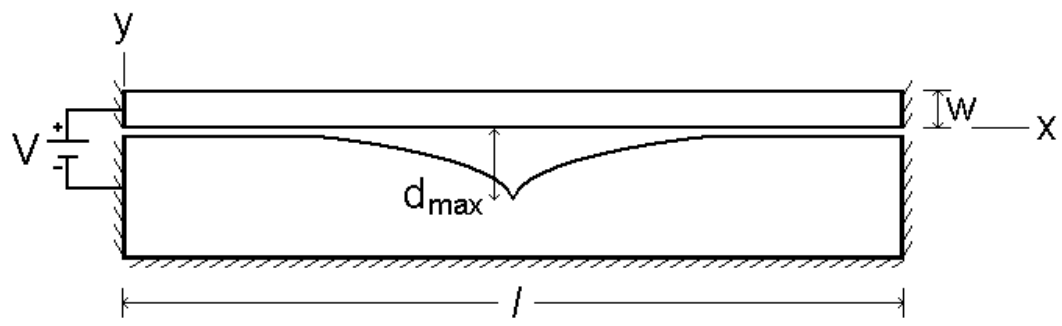

(b)

Table 7. Polynomial order and corresponding pull-in voltage for cantilever and fixed-fixed beams (required $2 \mu \mathrm{m}$ displacement).

\begin{tabular}{ccccc}
\hline$n$ & $\begin{array}{c}\mathbf{V}_{\text {PI }}[\mathbf{V}] \\
\text { cantilever }\end{array}$ & Gap Width $\left(\boldsymbol{d}_{\text {max }}\right)[\boldsymbol{\mu m}]$ cantilever & $\begin{array}{c}\mathbf{V}_{\text {PI }}[\mathbf{V}] \\
\text { fixed-fixed }\end{array}$ & $\begin{array}{c}\text { Gap Width }\left(\boldsymbol{d}_{\text {max }}\right)[\boldsymbol{\mu m}] \\
\text { fixed-fixed }\end{array}$ \\
\hline 0.9166 & ------- & ------- & 126.24 & 6.18 \\
1.0000 & 14.86 & 4.84 & 125.48 & 6.57 \\
1.0833 & ------- & ------ & 126.63 & 7.08 \\
1.1666 & 14.26 & 5.17 & 128.91 & 7.71 \\
1.2916 & 14.15 & 5.55 & ------- & ----- \\
1.3333 & 14.14 & 5.69 & 137.68 & 9.53 \\
1.3750 & 14.22 & 5.88 & ------- & ----- \\
1.5000 & 14.43 & 6.49 & 151.20 & 12.30 \\
1.6666 & 14.97 & 7.57 & ------- & ------ \\
\hline
\end{tabular}

Figure 11. Pull-in voltages for polynomial gap cases (a) cantilever beam (b) fixed-fixed beam.

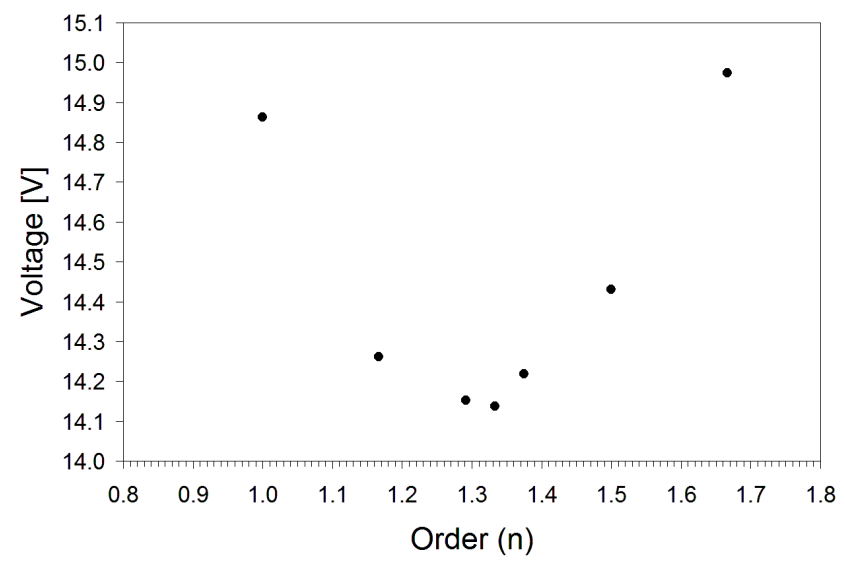

(a)

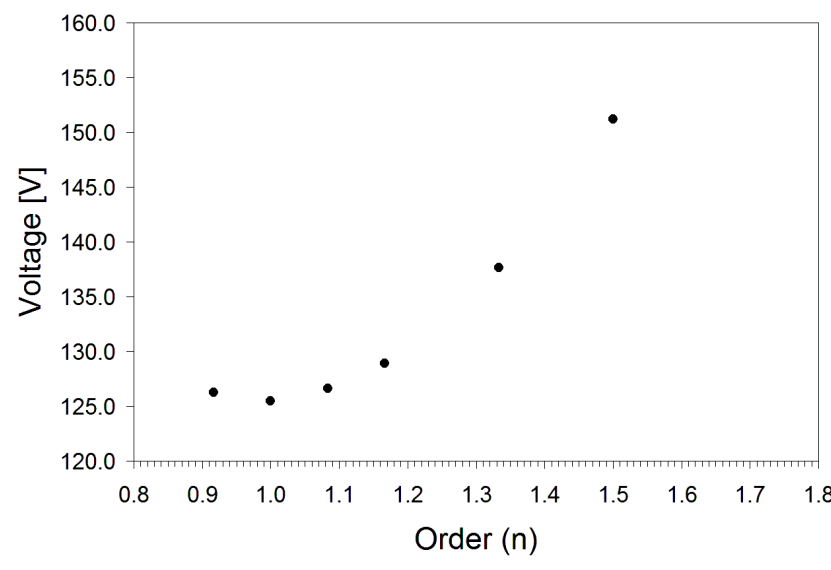

(b) 
Figure 12. Cantilever beam polynomial gap $(n=1.3333)$ beam displacement profile.

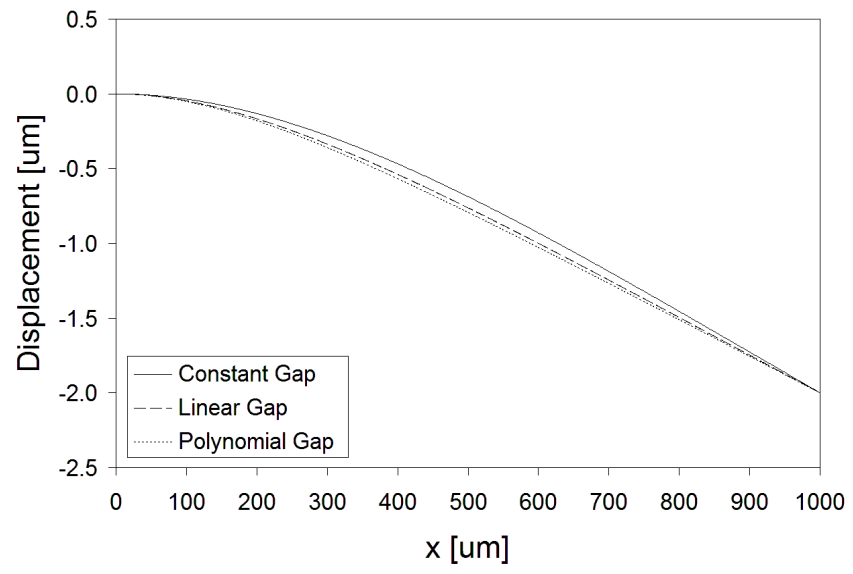

\section{Conclusions}

Significant reductions in the pull-in voltage of both cantilever beam structures and fixed-fixed beam structures have been made by varying the geometry of the air gap while still achieving a required actuator displacement. The finite element simulator ANSYS Multiphysics has been used to determine both the pull-in voltage and the displacement of the beams before pull-in occurs. These simulations have been verified against both theoretical equations for pull-in voltage as well as previously published results from an independent finite element simulator and the results agree to better than $1 \%$ on average in all cases. Significant reductions in pull-in voltage can be made while still maintaining required displacement by simply varying the gap profile in a linear fashion. In the case of the cantilever beam this results in the pull-in voltage being reduced by $37.2 \%$ and for the fixed-fixed beam case the reduction is $29.6 \%$. Slightly more complicated shapes can provide a further reduction in pull-in voltage. For the cantilever beam a polynomial $(n=4 / 3)$ shape provides a further $4.8 \%$ reduction in pull-in voltage and by flattening the bottom of the linearly varying gap in the fixed-fixed beam case a further $1.2 \%$ reduction in pull-in voltage can be obtained. These simple electrode shapes, while not necessarily optimal, provide significantly large reductions in pull-in voltage, while still maintaining the same maximum beam displacement. In addition, the average beam displacement has increased, which can be important in applications such as RF MEMS variable capacitors. Further reductions obtained from optimal electrode shapes are believed to be minimal.

\section{References}

1. Klymyshyn, D.M.; Haluzan, D.T.; Börner, M.; Achenbach, S.; Mohr, J.; Mappes, T. High aspect ratio vertical cantilever RF-MEMS variable capacitor. IEEE Microw. Wireless Compon. Lett. 2007, 17, 127-129.

2. Jung, S.; Kang, K.; Park, J.-H.; Chung, K.-W.; Kim, Y.-K.; Kwon, Y. Micromachined frequency-variable impedance tuners using resonant unit cells. IEEE MTT-S Int. Microw. Symp. Dig. 2001, 1, 333-336.

3. Goldsmith, C; Randall, J.; Eshelman, S.; Lin, T.H.; Denniston, D.; Chen, S.; Norvell, B. Characteristics of micromachined switches at microwave frequencies. IEEE MTT-S Int. Microw. Symp. Dig. 1996, 2, 1141-1144. 
4. Hung, E.S.; Senturia, S.D. Extending the travel range of analog-tuned electrostatic actuators. $J$. Microelectromech. Syst. 1999, 8, 497-505.

5. Huff, M.A.; Mettner, M.S.; Lober, T.A.; Schmidt, M.A. A pressure-balanced electrostatically-actuated microvalve. Tech. Dig. IEEE Solid-State Sens. Actuator Workshop 1990, 1, 123-127.

6. Legtenberg, R.; Gilbert, J.; Senturia, S.D.; Elwenspoek, M. Electrostatic curved electrode actuators. J. Microelectromech. Syst. 1997, 6, 257-265.

7. Najar, F.; Choura, S.; El-Borgi, S.; Abdel-Rahman, E.M.; Nayfeh, A.H. Modeling and design of variable-geometry electrostatic microactuators. J. Micromech. Microeng. 2005, 15, 419-429.

8. Abdalla, M.M.; Reddy, C.K.; Faris, W.F.; Gurdal, Z. Optimal design of an electrostatically actuated microbeam for maximum pull-in voltage. Comput. Struct. 2005, 83, 1320-1329.

9. Becker, E.W.; Ehrfeld, W.; Hagmann, P.; Maner, A.; Münchmeyer, D. Fabrication of microstructures with high aspect ratios and great structural heights by synchrotron radiation lithography, galvanoforming, and plastic moulding (LIGA process). Microelectron. Eng. 1986, 4, 35-56.

10. ANSYS Multiphysics Version 11.0; ANSYS, Inc.: Canonsburg, PA, USA, 2006.

11. Osterberg, P.M. Electrostatically actuated microelectromechanical test structures for material property measurements. Ph.D. Thesis, Massachusetts Institute of Technology, Cambridge, MA, USA, 1995.

12. Osterberg, P.M.; Senturia, S.D. M-TEST: A test chip for MEMS material property measurement using electrostatically actuated test structures. J. Microelectromech. Syst. 1997, 6, 107-118.

13. Chowdhury, S.; Ahmadi, M.; Miller, W.C. A closed-form model for the pull-in voltage of electrostatically actuated cantilever beams. J. Micromech. Microeng. 2005, 15, 756-763.

14. Chowdhury, S.; Ahmadi, M.; Miller, W.C. Pull-in voltage study of electrostatically actuated fixed-fixed beams using a VLSI on-chip interconnect capacitance model. J. Microelectromech. Syst. 2006, 15, 639-651.

15. Sadeghian, H.; Rezazadeh, G.; Osterberg, P.M. Application of the generalized differential quadrature method to the study of pull-in phenomena of MEMS switches. J. Microelectromech. Syst. 2007, 16, 1334-1340.

(C) 2010 by the authors; licensee MDPI, Basel, Switzerland. This article is an Open Access article distributed under the terms and conditions of the Creative Commons Attribution license (http://creativecommons.org/licenses/by/3.0/). 